Rev. Int. Contam. Ambie. 37, 539-551, 2021

https://doi.org/10.20937/RICA.53915

\title{
CALIDAD AMBIENTAL DE LA LAGUNA MARÍA LA GORDA DE LA CIUDAD DE RÍO GALLEGOS, ARGENTINA
}

Environmental quality of the María la Gorda lagoon in the city of Río Gallegos, Argentina

\author{
Adrián Javier ACUÑA ${ }^{1 *}$, Javier Américo SZEWCZUK ${ }^{1}$ y Graciela Natalia PUCCI ${ }^{2}$
}

\author{
${ }^{1}$ Grupo de Estudios Ambientales, Facultad Regional Santa Cruz, Universidad Tecnológica Nacional, Avenida \\ de los Inmigrantes 555, Río Gallegos, Santa Cruz, Argentina \\ ${ }^{2}$ Centro de Estudios e Investigaciones en Microbiología Aplicada, Universidad Nacional de la Patagonia San \\ Juan Bosco, Ciudad Universitaria, Comodoro Rivadavia, Chubut, Argentina \\ *Autor para correspondencia: adrianjacuna@yahoo.com.ar
}

(Recibido: marzo de 2020; aceptado: diciembre de 2020)

Palabras claves: laguna, reserva urbana, calidad, ambiente, recreación.

\section{RESUMEN}

La laguna María la Gorda forma parte del Sistema de Reserva Natural Urbana de la ciudad de Río Gallegos, siendo una de las de mayor superficie con aproximadamente 11.29 ha. El objetivo de este trabajo fue caracterizar desde el punto de vista físico, químico, microbiológico y de contaminantes específicos la calidad del agua y el sedimento de la laguna María la Gorda con el fin de conocer su calidad ambiental. Para ello se tomaron muestras de agua y sedimento de forma estacional y se estudiaron en función de sus características físicas, químicas, microbiológicas, así como de su concentración de hidrocarburos y metales pesados. Los principales resultados obtenidos demostraron que el agua de la laguna María la Gorda tiene calidad ambiental media. $\mathrm{Su}$ uso debe limitarse a fines recreativos debido a la presencia de contaminación por materia orgánica con elevados valores de DQO, nitratos y microorganismos indicadores; sin embargo, no se encontraron presentes hidrocarburos ni metales pesados por encima de los valores de referencia utilizados. La demostración de la deficiente calidad de este espacio catalogado como reserva urbana obliga a implantar medidas que tiendan al saneamiento y mantenimiento de la buena calidad ambiental de la zona de estudio, a fin de darle el uso para el cual está destinada y evitar que, en el futuro, surjan inconvenientes ambientales que lleven a la aparición de problemas de salud en la población de Río Gallegos asociados con la problemática planteada.

Keywords: lagoon, urban reserve, quality, environment, recreation.

\begin{abstract}
The María la Gorda lagoon is part of the Urban Natural Reserve System of the city of Río Gallegos, being one of the largest reserve areas, with approximately 11.29 ha. The objective of this work was to characterize the quality of water and sediment in the María la Gorda lagoon from a physical, chemical, microbiological and specific-pollutant point of view, in order to know its environmental quality. For this, water and sediment samples were taken seasonally and studied according to their physical, chemical, and
\end{abstract}


microbiological characteristics, as well as their concentration in hydrocarbons and heavy metals. The main results obtained showed that the environmental quality of the María la Gorda lagoon's water is average, and it should only be used for recreational purposes due to the presence of organic matter with high COD values, nitrates and indicator microorganisms; however, no hydrocarbons or heavy metals were present above the reference values. The evidence of poor environmental quality in the study area, classified as an urban reserve, makes it necessary to implement sanitizing measures which tend to maintain a good environmental quality, so that it may continue to serve its intended purpose without future environmental problems. These could lead to emerging health problems in the population of Río Gallegos associated with the problem raised.

\section{INTRODUCCIÓN}

La ciudad de Río Gallegos está ubicada en el extremo sudeste de la región patagónica continental, en la provincia de Santa Cruz, Argentina, y está asentada sobre la margen sur del estuario del río Gallegos. Fue construida sobre materiales aluviales depositados en épocas de deshielo glacial donde la disponibilidad hídrica era de gran magnitud. Desde 2004 Río Gallegos cuenta con su primera reserva costera urbana, creada por el decreto núm. 3478/06 del Poder Ejecutivo Municipal. Así se generó el primer precedente de protección de ambientes intermareales en un municipio de la Patagonia para la conservación de paisajes terrestres y marinos con fines recreativos, de acuerdo con las directrices establecidas por la Unión Internacional para la Conservación de la Naturaleza. En 2009, fortaleciendo el antecedente de 2004, el Honorable Concejo Deliberante de Río Gallegos creó el Sistema de Reservas Naturales Urbanas (SRNU) de la ciudad mediante la ordenanza núm. 6762/09. En dicho sistema se incluyen cuatro lagunas urbanas que se utilizan mayormente para educación ambiental y esparcimiento de la población.

Las reservas urbanas son espacios naturales o modificados, continentales o marinos, que poseen biodiversidad, valores naturales y/o culturales. Son de interés principalmente local, prestan servicios ecosistémicos y son reconocidos por el municipio local mediante un instrumento legal, en concordancia con el sistema nacional de áreas protegidas y las políticas ambientales, territoriales y de desarrollo municipal y nacional (Ferrari et al. 2014). La importancia de este sistema de reservas radica en la conservación del paisaje natural. En el caso de los humedales, estos constituyen un medio de almacenamiento de aguas meteóricas que impide la inundación de sectores aledaños a la ciudad; a su vez, ofrecen un hábitat para especies de fauna silvestre, propiciando un ambiente cálido y natural para el desarrollo de actividades de educación ambiental y prácticas de campo con estudiantes de diferentes niveles educativos; sumado a esto, brindan un espacio ideal para alentar el turismo de naturaleza mediante el avistamiento de aves autóctonas (Decristófaro et al. 2014) y el esparcimiento de la población.

El SRNU cuenta con estudios geológicos, de biodiversidad de flora y de fauna actualizados; sin embargo, no hay datos preexistentes respecto a la calidad del agua y sedimentos de sus lagunas. Esto ocasiona una situación delicada, ya que dichas lagunas son utilizadas principalmente con fines recreativos por los pobladores de Río Gallegos.

El estudio de la calidad de agua y sedimentos de la laguna María la Gorda (LMG) tendrá un gran impacto desde el punto de vista ambiental y económico, ya que se podrá contar con un conocimiento exhaustivo de su estado de contaminación. Esto servirá para direccionar de forma adecuada los recursos disponibles para tareas de saneamiento, en caso de ser necesario, lo que redundará en un ambiente más saludable para la población que desee realizar diferentes actividades en estos sitios protegidos.

El objetivo de este trabajo fue caracterizar desde el punto de vista físico, químico, microbiológico y de contaminantes específicos la calidad del agua y el sedimento de la LMG de la ciudad de Río Gallegos, Santa Cruz, Argentina, con el fin de conocer su calidad ambiental.

\section{MATERIALES Y MÉTODOS}

\section{Descripción del área de estudio}

La LMG forma parte del SRNU de la ciudad de Río Gallegos, siendo una de las de mayor superficie con aproximadamente $11.29 \mathrm{ha}$. Se localiza en el interior de la ciudad ( $51^{\circ} 37$ '31.38" S-69 14 ' 31.53” O) y protege un humedal natural que brinda invaluables servicios ambientales a los vecinos de la comunidad. Actúa como centro natural de filtrado urbano y depó- 
sito de precipitaciones. La riqueza máxima de aves registrada fue de 34 especies, entre las que destacan numerosas aves acuáticas como cisnes, patos y macaes (Podiceps spp.), además de playeras (Ferrari y Montero 2015). Actualmente la laguna se encuentra dividida en dos cuerpos de agua (Fig. 1), de los cuales el de menor tamaño (Sec1) ha sido totalmente modificado para la realización de actividades recreativas como ciclismo, caminatas, juegos de niños, etc. El de mayor tamaño (Sec2) suele utilizarse para esparcimiento, con actividades como práctica del kayak, educación ambiental y otras; al estar menos modificado, las aves suelen preferirlo para descansar y alimentarse. El eje longitudinal de la Sec1 mide aproximadamente 160 $\mathrm{m}$, con un ancho promedio de $46 \mathrm{~m}$. Por su parte, la Sec2 tiene un largo aproximado de $515 \mathrm{~m}$, con un ancho promedio de $120 \mathrm{~m}$. Ambas secciones tienen una profundidad aproximada un poco superior a $1 \mathrm{~m}$ en su parte más profunda.

\section{Toma de muestras}

Para la realización de los estudios propuestos se seleccionaron ocho puntos de muestreo en LMG según lo mostrado en la figura 1 y el cuadro I. En las ocho estaciones de muestreo se recolectaron muestras de agua y sedimento durante las cuatro estaciones del año. Las muestras se recolectaron a una distancia de 3-4 m de la línea de costa en botellas color ámbar de un litro de capacidad para los análisis físicos y químicos, así como de hidrocarburos y metales pesados, y en un recipiente estéril de $250 \mathrm{~mL}$ para los estudios microbiológicos. Aproximadamente $1 \mathrm{~kg}$ de sedimento se tomó en cada punto de muestreo y se dejó escurrir para la eliminación del agua libre; finalmente se almacenó en bandejas de plástico nuevas. Las muestras tomadas en una misma estación fueron analizadas de forma individual y los resultados
CUADRO I. COORDENADAS GEOGRÁFICAS DE LOS SITIOS DE MUESTREO SELECCIONADOS EN LA LAGUNA MARÍA LA GORDA DE LA CIUDAD DE RÍO GALLEGOS.

\begin{tabular}{ccc}
\hline Muestra & Latitud & Longitud \\
\hline M1 & $51^{\circ} 37^{\prime} 28.11^{\prime \prime}$ & $69^{\circ} 14^{\prime} 24.61^{\prime \prime}$ \\
M2 & $51^{\circ} 37^{\prime} 31.38^{\prime \prime}$ & $69^{\circ} 14^{\prime} 31.53^{\prime \prime}$ \\
M3 & $51^{\circ} 37^{\prime} 33.67^{\prime \prime}$ & $69^{\circ} 14^{\prime} 40.24^{\prime \prime}$ \\
M4 & $51^{\circ} 37^{\prime} 34.88^{\prime \prime}$ & $69^{\circ} 14^{\prime} 48.43^{\prime \prime}$ \\
M5 & $51^{\circ} 37^{\prime} 30.96^{\prime \prime}$ & $69^{\circ} 14^{\prime} 44.45^{\prime \prime}$ \\
M6 & $51^{\circ} 37^{\prime} 28.25^{\prime \prime}$ & $69^{\circ} 14^{\prime} 33.78^{\prime \prime}$ \\
M7 & $51^{\circ} 37^{\prime} 27.13^{\prime \prime}$ & $69^{\circ} 14^{\prime} 17.78^{\prime \prime}$ \\
M8 & $51^{\circ} 37^{\prime} 24.99^{\prime \prime}$ & $69^{\circ} 14^{\prime} 12.59^{\prime \prime}$ \\
\hline
\end{tabular}

expresados representan los promedios obtenidos. Todas las muestras se mantuvieron refrigeradas hasta su análisis.

En el momento de la recolección de las muestras de agua se realizó la determinación in situ del $\mathrm{pH}$, conductividad, temperatura, y sólidos y oxígeno disueltos. Para la determinación de $\mathrm{pH}$, conductividad, temperatura y sólidos disueltos se utilizó un equipo marca Hanna modelo HI991301 equipado con sonda multiparamétrica de la misma marca (modelo HI1288). Para la determinación de oxígeno disuelto se utilizó un equipo marca APERA modelo DO850 equipado con una sonda modelo DO803.

\section{Estudios físicos y químicos}

Se realizaron estudios físicos y químicos de las muestras de agua. El carbonato y bicarbonato se analizaron mediante titulación con ácido clorhídrico $0.1 \mathrm{~N}$ utilizando fenolftaleína y heliantina como indicadores. El calcio se determinó por complejometría con EDTA y murexida como indicador a $\mathrm{pH} 12$ y el magnesio con negro de eriocromo $\mathrm{T}$ como indicador

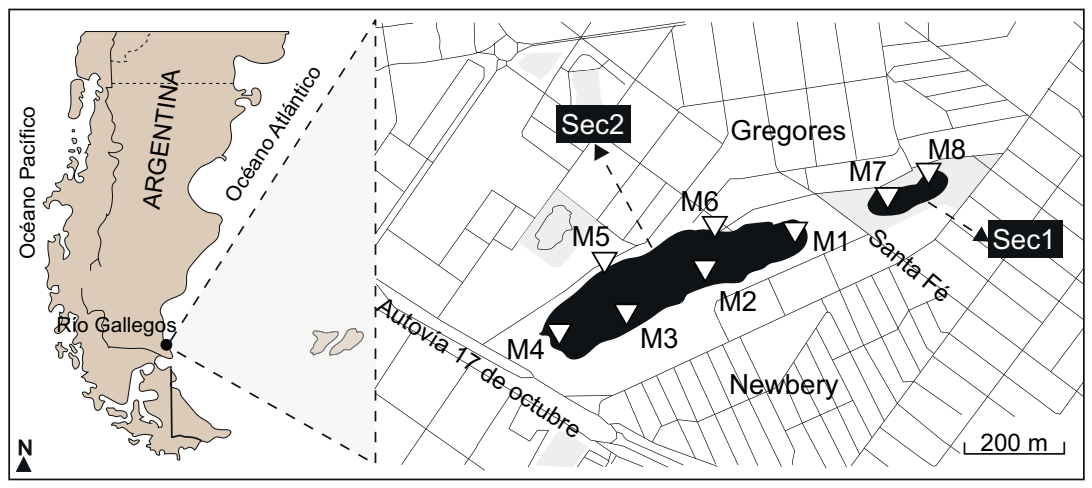

Fig. 1. Distribución espacial de las muestras recolectadas en la laguna María la Gorda de la ciudad de Río Gallegos. 
a pH 10. El ion cloruro se determinó por el método de Mohr, el ion amonio con azul de indofenol y el ion fosfato con azul de molibdeno. El nitrito se determinó colorimétricamente con ácido sulfanílico y 1-naftilamina y el nitrato con brucina en presencia de ácido sulfúrico. El sodio y el potasio se determinaron por fotometría de llama y el contenido de sulfato se estableció mediante el método turbidimétrico basado en su precipitación en un medio ácido. En las muestras de agua también se estudiaron la demanda biológica de oxígeno (DBO) por el método automatizado con sensor de presión, la demanda química de oxígeno (DQO) por digestión ácida a $160{ }^{\circ} \mathrm{C}$ y la turbidez por el método nefelométrico. Los sólidos totales se determinaron por gravimetría a $105{ }^{\circ} \mathrm{C}$. Todas las determinaciones analíticas se realizaron de acuerdo con lo propuesto por Rice et al. (2017).

\section{Análisis de hidrocarburos}

La determinación de hidrocarburos totales del petróleo (HTP) se realizó en las muestras de agua por aplicación del método EPA 418.1 (US-EPA 1978), y en las de sedimento por aplicación del método EPA 3550C/418.1 (US-EPA 2007, US-EPA 1978).

\section{Análisis de metales pesados}

Antes de la cuantificación, las muestras de sedimentos $(0.5 \mathrm{~g})$ fueron sometidas a una extracción con ácido nítrico según lo establecido en la norma EPA 3050B (US-EPA 1996). Los metales Ag, As, Cd, $\mathrm{Cu}, \mathrm{Cr}, \mathrm{Hg}$, Ni, Pb, Zn, Ba y Se cuantificaron por espectrometría de absorción atómica según las normas EPA 7000B (US-EPA 2007) tanto en las muestras de agua como en las de sedimento.

\section{Análisis microbiológico}

La cuantificación de bacterias coliformes totales (CT) y fecales (CF) se evaluó por el método del número más probable (NMP) utilizando caldo MacConkey e incubación a 35 y $44{ }^{\circ} \mathrm{C}$, respectivamente. La presencia de $E$. coli se confirmó por identificación con las pruebas IMVIC (indol, rojo de metilo, Voges Proskauer y citrato). Las cuantificaciones de bacterias heterotróficas totales (BAT) y Pseudomonas aeruginosa se realizaron en placa, la primera empleando el método de diseminación en superficie con incubación a $37^{\circ} \mathrm{C}$ durante $48 \mathrm{~h}$ en agar nutritivo, y la segunda con el método de diseminación en superficie en agar cetrimida e incubación a $37^{\circ} \mathrm{C}$ por $48 \mathrm{~h}$. El género Enterococos se cuantificó por el método de NMP utilizando agar bilis esculina. Todas las determinaciones propuestas se realizaron de acuerdo con lo propuesto por Rice et al. (2017).
Para evaluar el estado de contaminación general se determinaron varios índices de calidad ambiental. A partir de los datos físicos, químicos y microbiológicos obtenidos en las muestras de agua se calcularon los índices de calidad de agua (ICA), de contaminación por mineralización (ICOMI) y de contaminación por materia orgánica (ICOMO) (Ramírez et al. 1997). El ICA fue calculado como la suma de nueve parámetros (oxígeno disuelto, DBO, nitratos, fósforo total, sólidos totales disueltos, turbidez, coliformes fecales, $\mathrm{pH}$ y diferencia entre temperatura ambiente y temperatura del agua) multiplicados por un valor atribuido en función de la importancia del parámetro, tomando un valor entre 0 y 100 adimensional, según la ecuación 1:

$\mathrm{ICA}=\sum_{i=1}^{n} W_{i} Q_{i}$

Para calcular el índice se utilizaron los factores de ponderación $\left(W_{\mathrm{i}}\right)$ propuestos por la National Sanitation Fundation (NSF) para cada parámetro, y factores de escala $\left(Q_{\mathrm{i}}\right)$ calculados mediante ajustes polinómicos a las curvas de estandarización asociadas con cada variable, formulados por la NSF (Elordi et at. 2013).

El ICOMO fue calculado en función a tres variables que incluyeron $\mathrm{DBO}$, coliformes totales y el porcentaje de saturación de oxígeno (ecuación 2):

$\mathrm{ICOMO}=1 / 3\left(I_{D B O}+I_{C T}+I_{O D \%}\right)$

donde $I_{\mathrm{DBO}}=-0.05+0.7 \log \mathrm{DBO}(\mathrm{mg} / \mathrm{L}) ; I_{\mathrm{CT}}=-1.44$ $+0.56 \log \mathrm{CT}(\mathrm{NMP} / 100 \mathrm{~mL}), \mathrm{y} I_{\mathrm{OD}} \%=1-0.01 \mathrm{OD}$ (\% sat) (Elordi et at. 2013).

Finalmente, el ICOMI fue calculado utilizando los resultados de las determinaciones de conductividad, dureza y alcalinidad de acuerdo con ecuación 3:

ICOMI $=1 / 3\left(I_{\text {Conductividad }}+I_{\text {Dureza }}+I_{\text {Alcalinidad }}\right)$

donde $I_{\text {Conductividad }}=10^{\log I_{\text {Conduct }}}\left(\log I_{\text {Conduct }}=\right.$ $-3.26+1.34 \log$ conductividad $(\mu \mathrm{S} / \mathrm{cm})) ; I_{\text {Dureza }}=$ $10^{\log I_{\text {Dureza }}\left(\log I_{\text {Dureza }}=-9.09+4.40 \log \text { dureza }\right.}$ $(\mathrm{mg} / \mathrm{L}))$ y $I_{\text {Alcalinidad }}=-0.25+0.005$ alcalinidad $(\mathrm{mg} / \mathrm{L}))$ (Ramírez et al. 1997).

Por otro lado, para conocer las características del agua para su potencial uso para riego, se realizaron análisis de Piper y Riverside utilizando el programa Diagrammes 6.48.

\section{Análisis de resultados}

Los resultados obtenidos se sometieron a un análisis de varianza (ANOVA). Se realizó un estudio de 
estadística multivariada de componentes principales (PCA) sobre el reordenamiento de los datos fisicoquímicos en una matriz de correlación utilizando el programa PAST 4.02 (Hammer et al. 2001).

\section{RESULTADOS Y DISCUSIÓN}

\section{Caracterización física y química}

$\mathrm{El} \mathrm{pH}$ representa el grado de acidez o alcalinidad del agua $\mathrm{y}$, de tal forma, interviene en las formas y procesos químicos e impactos ambientales de numerosas sustancias presentes en la misma (EPA 2013). Además de su importancia para la biota, el pH también es un indicador de contaminación. En LMG el pH se mantuvo cercano a 9 en casi todas las estaciones del año, con la excepción del verano, cuando fluctuó entre valores significativamente mayores de alrededor de $11(\mathrm{p}<0.05)$ (Cuadro II). La dinámica temporal del $\mathrm{pH}$ puede ofrecer una importante variabilidad estacional e incluso diaria en cursos naturales de agua. La fotosíntesis realizada por organismos acuáticos aumenta el consumo de $\mathrm{CO}_{2}$, causando incremento en el $\mathrm{pH}$. Rubio-Arias et al. (2016) estudiaron esta situación en el lago Colina de la ciudad de Chihuahua, México, y atribuyeron los incrementos de $\mathrm{pH}$ observados a la fotosíntesis realizada por algas que se desarrollaron por exceso de volcados de efluentes cloacales. Durante la estación de verano se observó en la LMG el cambio más significativo en la temperatura del agua $(\mathrm{p}<0.05)$ con un valor de $15.35 \pm 0.52{ }^{\circ} \mathrm{C}$. También se encontró la mayor concentración de carbonatos $(\mathrm{p}<0.05)$ y la menor cantidad de bicarbonato cuantificada $(\mathrm{p}<0.05)$ durante el año de estudio. Estos cambios observados en la concentración de carbonatos y bicarbonatos, sumados a una posible disminución del $\mathrm{CO}_{2}$ del agua por un proceso fotosintético, pueden ser los responsables de los altos valores de $\mathrm{pH}$ encontrados en la LMG, principalmente en verano (Weiner 2012), ya que la presencia de compuestos nitrogenados (nitrato) y fosforados (fosfato) tienden a estimular los procesos de eutrofización y acelerar el desarrollarlo de organismos acuáticos que pueden realizar estos procesos biológicos.

Sin bien en el presente estudio no se realizó la determinación de clorofila $a$, si se evidenció la presencia de macroalgas en la estación de verano en los trabajos de muestreo realizados. Dado que uno de los principales usos de la LMG es recreacional, el agua recreativa no debe poseer valores de $\mathrm{pH}$ muy bajos o altos, ya que según la Organización Mundial de la Salud esto puede causar problemas en la piel y los ojos por su contacto (WHO 2003). En los estándares de calidad del agua recreativa de la Agencia de Protección Ambiental de Estados Unidos (US-EPA), se establece como rango óptimo de $\mathrm{pH}$ para esta actividad el comprendido entre 6.5 y 8.5 (US-EPA 2012). En el cuadro II se puede observar que los valores de DQO fueron altos en todas las estaciones del año, acompañados de altas concentraciones de compuestos nitrogenados y fosfatos. Respecto de la DBO, sus valores máximos se observaron en primavera y otoño con promedios de $5.9 \pm 11$ y $5.2 \pm 8.1 \mathrm{mg} / \mathrm{L}$, respectivamente. Los valores mínimos de $\mathrm{DBO}$ encontrados fueron de $<0.1 \mathrm{mg} / \mathrm{L}$ y el máximo de $25.3 \mathrm{mg} / \mathrm{L}$ $(\mathrm{p}<0.05)$ en primavera y de $<0.1$ y $18.6 \mathrm{mg} / \mathrm{L}(\mathrm{p}$ $<0.05$ ) en otoño, correspondiendo todos los valores mas bajos observados a la Sec2 de LMG, y los valores mas elevados al Sec1

La presencia de nitritos durante todas las estaciones del año es un indicador de la presencia de contaminación por materia orgánica reciente, ya que este compuesto es altamente inestable en agua. El nitrógeno puede aparecer en forma de amonio y por oxidación puede transformarse en nitrito y finalmente en nitrato, que es la forma más usual y estable (de Miguel-Fermández y Vázquez-Taset 2006). Dado que la presencia de amonio favorece la multiplicación microbiana, su detección en el agua en cantidades significativas se considera un indicio de probable contaminación reciente. En el caso particular del nitrato, su concentración promedio fue de aproximadamente $3 \mathrm{mg} / \mathrm{L}$ durante todo el año, con la excepción del otoño, cuando alcanzó valores significativamente mayores $(\mathrm{p}<0.05)$ de $6.03 \pm 6.39 \mathrm{mg} / \mathrm{L}(\sim 3 \mathrm{mg} / \mathrm{L}$ promedio en Sec2 y $15 \mathrm{mg} / \mathrm{L}$ en Sec1 $(\mathrm{p}<0.05))$.

El oxígeno disuelto es uno de los indicadores más importantes de la calidad de las aguas naturales para albergar vida en éstas y para establecer el grado de impacto del vuelco de materia orgánica (McCutcheon et al. 1993, Allan y Castillo 2007). En el caso de las determinaciones realizadas, se pudo observar que este parámetro se mantuvo en valores de 7.5 a $9.5 \mathrm{mg} / \mathrm{L}$ salvo en otoño, cuando los valores descendieron significativamente $(\mathrm{p}<0.05)$ a magnitudes cercanas a $6 \mathrm{mg} / \mathrm{L}$. Weiner (2008) establece que concentraciones de oxígeno disuelto en aguas naturales en el rango de 6.5 a $8 \mathrm{mg} / \mathrm{L}$ son características de aguas ligeramente contaminadas, siendo los valores $>$ a $8 \mathrm{mg} / \mathrm{L}$ los característicos de aguas libres de contaminación.

Estos compuestos encontrados en las aguas de la LMG son indicios importantes de su contaminación - al menos esporádica - con materia orgánica, lo cual puede estar relacionado con la presencia de desembocaduras de efluentes pluviales en las dos 
CUADRO II. DETERMINACIONES ANALÍTICAS REALIZADAS EN LAS MUESTRAS DE AGUA RECOLECTADAS EN LAS DIFERENTES ESTACIONES DEL AÑO.

\begin{tabular}{|c|c|c|c|c|}
\hline Parámetro & Invierno & Primavera & Verano & Otoño \\
\hline & \multicolumn{4}{|c|}{ Caracterización física y química } \\
\hline Temperatura $\left({ }^{\circ} \mathrm{C}\right)$ & $1.78 \pm 0.44$ & $5.58 \pm 1.12$ & $15.35 \pm 0.52$ & $8.20 \pm 0.55$ \\
\hline Oxígeno disuelto (mg/L) & $9.50 \pm 1.21$ & $7.86 \pm 1.07$ & $9.07 \pm 1.75$ & $6.57 \pm 1.13$ \\
\hline \% Saturación de oxígeno & $68 \pm 8$ & $62 \pm 8$ & $89 \pm 17$ & $55 \pm 9$ \\
\hline $\mathrm{pH}$ & $9.06 \pm 0.12$ & $8.59 \pm 0.46$ & $10.79 \pm 0.30$ & $8.74 \pm 0.24$ \\
\hline Conductividad $(\mu \mathrm{S} / \mathrm{cm})$ & $2135 \pm 298$ & $2036 \pm 287$ & $3043 \pm 589$ & $3815 \pm 1584$ \\
\hline Turbidez (UNT) & $21 \pm 24$ & $16 \pm 20$ & $14 \pm 20$ & $5 \pm 5$ \\
\hline Sólidos totales (mg/L) & $1536 \pm 184$ & $1464 \pm 178$ & $1600 \pm 209$ & $2304 \pm 965$ \\
\hline Sólidos en suspensión (mg/L) & $14 \pm 27$ & $14 \pm 25$ & $12 \pm 21$ & $<1$ \\
\hline Sólidos disueltos $(\mathrm{mg} / \mathrm{L})$ & $1525 \pm 213$ & $1454 \pm 205$ & $1564 \pm 215$ & $1904 \pm 791$ \\
\hline Alcalinidad (mg/L) & $362 \pm 47$ & $367 \pm 27$ & $419 \pm 57$ & $572 \pm 172$ \\
\hline Dureza $(\mathrm{mg} / \mathrm{L})$ & $104 \pm 4$ & $113 \pm 6$ & $118 \pm 4$ & $150 \pm 20$ \\
\hline $\mathrm{DBO}(\mathrm{mg} / \mathrm{L})$ & $<0.1$ & $5.9 \pm 11$ & $3.4 \pm 5$ & $5.2 \pm 8.1$ \\
\hline DQO (mg/L) & $266 \pm 35$ & $288 \pm 41$ & $317 \pm 20$ & $378 \pm 170$ \\
\hline Calcio (mg/L) & $20 \pm 1$ & $20 \pm 1$ & $22 \pm 4$ & $24 \pm 9$ \\
\hline Magnesio (mg/L) & $13 \pm 1$ & $15 \pm 1$ & $15 \pm 2$ & $22 \pm 8$ \\
\hline Sodio $(\mathrm{mg} / \mathrm{L})$ & $581 \pm 116$ & $562 \pm 87$ & $687 \pm 148$ & $450 \pm 187$ \\
\hline Potasio (mg/L) & $13 \pm 2$ & $15 \pm 1$ & $15 \pm 1$ & $22 \pm 5$ \\
\hline Carbonato (mg/L) & $<1$ & $<1$ & $155 \pm 51$ & $<1$ \\
\hline Bicarbonato (mg/L) & $443 \pm 58$ & $447 \pm 33$ & $197 \pm 34$ & $698 \pm 210$ \\
\hline Cloruro $(\mathrm{mg} / \mathrm{L})$ & $700 \pm 110$ & $724 \pm 110$ & $822 \pm 162$ & $987 \pm 463$ \\
\hline Sulfato (mg/L) & $2 \pm 1$ & $2 \pm 1$ & $4 \pm 2$ & $25 \pm 17$ \\
\hline Nitrato (mg/L) & $2.59 \pm 0.38$ & $3.01 \pm 1.42$ & $3.72 \pm 1.96$ & $6.03 \pm 6.39$ \\
\hline Nitrito (mg/L) & $0.06 \pm 0.12$ & $0.15 \pm 0.03$ & $0.05 \pm 0.08$ & $0.35 \pm 0.52$ \\
\hline Amonio (mg/L) & $0.10 \pm 0.06$ & $0.82 \pm 1.01$ & $0.03 \pm 0.06$ & $0.26 \pm 0.26$ \\
\hline \multirow[t]{2}{*}{ Fosfato (mg/L ) } & $0.22 \pm 0.03$ & $0.41 \pm 0.16$ & $0.16 \pm 0.29$ & $0.57 \pm 0.14$ \\
\hline & \multicolumn{4}{|c|}{ Caracterización microbiológica } \\
\hline BAT (UFC/mL) & $559 \pm 748$ & $1316 \pm 692$ & $415 \pm 199$ & $1499 \pm 970$ \\
\hline CT (NMP/100mL) & $18 \pm 26$ & $552 \pm 491$ & $19 \pm 29$ & $197 \pm 365$ \\
\hline $\mathrm{CF}(\mathrm{NMP} / 100 \mathrm{~mL})$ & $5 \pm 8$ & $88 \pm 181$ & $3 \pm 5$ & $<3$ \\
\hline E. coli $(\mathrm{NMP} / 100 \mathrm{~mL})$ & $5 \pm 8$ & $11 \pm 25$ & $<3$ & $<3$ \\
\hline$P$. aeruginosa $(\mathrm{UFC} / \mathrm{mL})$ & $<10$ & $<10$ & $<10$ & $<10$ \\
\hline \multirow[t]{2}{*}{ Enterococos (NMP/100mL) } & $5 \pm 8$ & $148 \pm 223$ & $<3$ & $11 \pm 10$ \\
\hline & \multicolumn{4}{|c|}{ Contaminantes específicos } \\
\hline $\operatorname{HTP}(\mathrm{mg} / \mathrm{L})$ & $<0.5$ & $<0.5$ & $<0.5$ & $<0.5$ \\
\hline Arsénico $(\mu \mathrm{g} / \mathrm{L})$ & $<2$ & $<2$ & $<2$ & $<2$ \\
\hline Bario $(\mathrm{mg} / \mathrm{L})$ & $<0.1$ & $<0.1$ & $<0.1$ & $<0.1$ \\
\hline Cadmio $(\mu \mathrm{g} / \mathrm{L})$ & $<5$ & $<5$ & $<5$ & $<5$ \\
\hline Cinc $(\mathrm{mg} / \mathrm{L})$ & $<0.1$ & $<0.1$ & $<0.1$ & $<0.1$ \\
\hline Cobre $(\mathrm{mg} / \mathrm{L})$ & $<0.1$ & $<0.1$ & $<0.1$ & $<0.1$ \\
\hline Cromo $(\mathrm{mg} / \mathrm{L})$ & $<0.05$ & $<0.05$ & $<0.05$ & $<0.05$ \\
\hline Mercurio $(\mu \mathrm{g} / \mathrm{L})$ & $<1$ & $<1$ & $<1$ & $<1$ \\
\hline Níquel $(\mu \mathrm{g} / \mathrm{L})$ & $<5$ & $<5$ & $<5$ & $<5$ \\
\hline Plata (mg/L) & $<0.01$ & $<0.01$ & $<0.01$ & $<0.01$ \\
\hline Plomo (mg/L) & $<0.05$ & $<0.05$ & $<0.05$ & $<0.05$ \\
\hline Selenio $(\mu \mathrm{g} / \mathrm{L})$ & $<4$ & $<4$ & $<4$ & $<4$ \\
\hline
\end{tabular}

DBO: demanda biológica de oxígeno, DQO: demanda química de oxígeno, BAT: bacterias aerobias totales, CT: coliformes totales, CF: coliformes fecales, HTP: hidrocarburos totales del petróleo 
secciones de la laguna. Estos efluentes tienen la función de recolectar el agua de lluvia de los sectores aledaños y canalizarla a su desembocadura en la LMG. Es importante destacar que de manera frecuente en los asentamientos aledaños suelen ocurrir desbordes de colectores cloacales, y los líquidos cloacales desbordados ingresan en el colector pluvial para finalmente ser vertidos en LMG.

En la figura 2 se presenta el análisis PCA realizado a partir de las características físicas y químicas del agua. En dicho análisis se puede observar claramente la tendencia observada en los datos presentados respecto de que ambas secciones de la LMG poseen características físicas y químicas diferentes, observándose que estas diferencias entre secciones son más acentuadas en verano y otoño. Al analizar la varianza total explicada, encontramos que el primer componente explica el $41.8 \%$ de la varianza, el segundo el $24.4 \%$ y el tercero el $12.7 \%$, llegándose a obtener con estos tres componentes el $78.9 \%$ de la
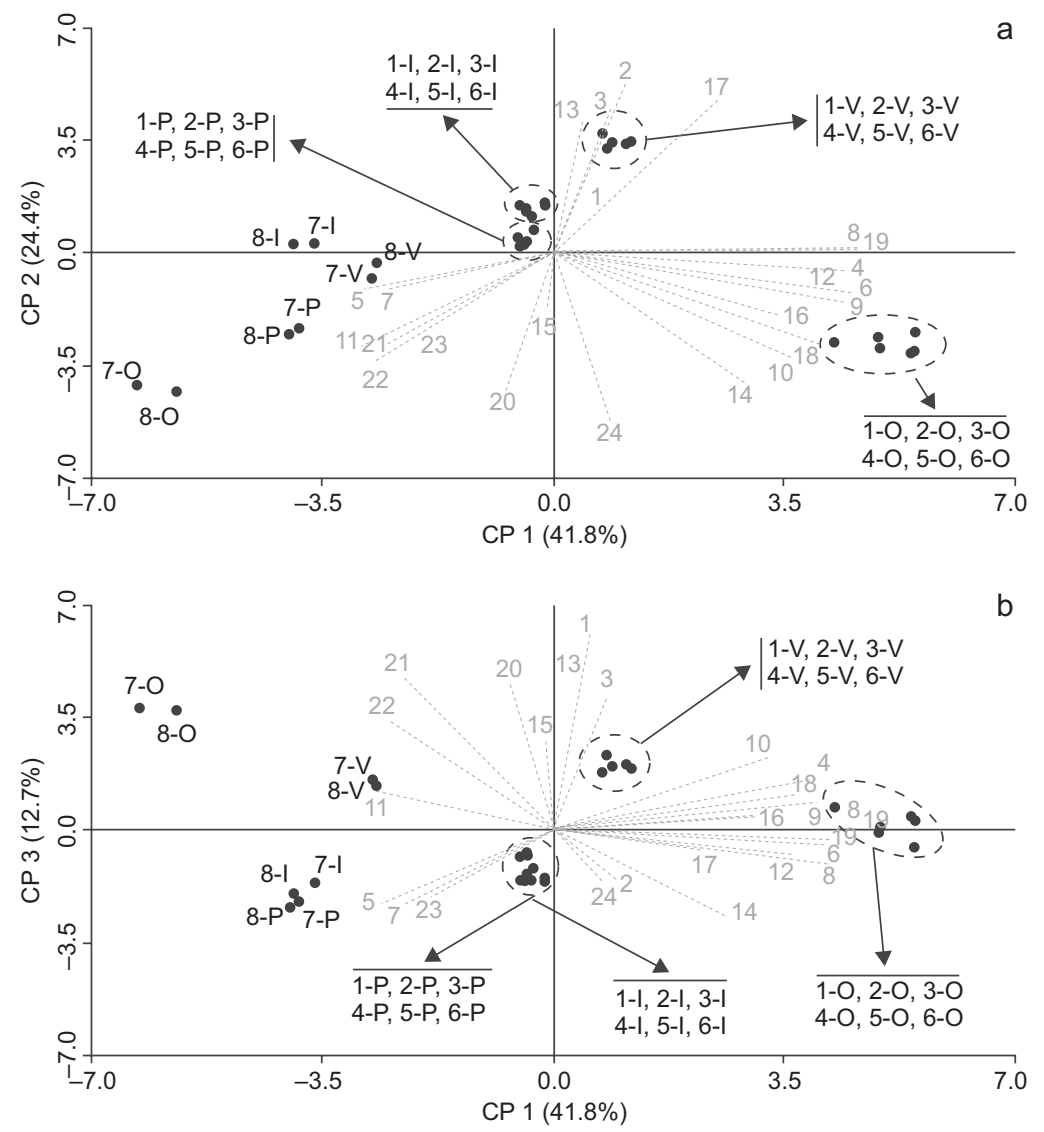

Fig. 2. Análisis de componentes principales (PCA) realizado en las muestras de agua respecto de los análisis físicos y químicos. En color gris se muestran los vectores de las variables que definen la construcción de los tres primeros componentes analizados. (a) Componentes 1 y 2 ; (b) componentes 1 y 3 ; (c) componentes 2 y 3 . Los números en negro representan el sitio de muestreo (I: invierno, P: primavera, V: verano, O: otoño). Los números en gris identifican el vector de cada variable (1: temperatura, 2 : oxígeno disuelto, $3: \mathrm{pH}, 4$ : conductividad, 5: turbidez, 6: sólidos totales, 7: sólidos en suspensión, 8: sólidos disueltos, 9: alcalinidad, 10: dureza, 11: DBO, 12: DQO, 13: carbonato, 14: bicarbonato, 15 : calcio, 16: magnesio, 17: sodio, 18: potasio, 19: cloruro, 20: sulfato, 21: nitrato: 22: nitrito, 23 amonio, 24: fosfato). 
varianza acumulada. De acuerdo con el cuadro III, las variables más correlacionadas en forma positiva con el primer componente fueron conductividad, solidos totales, sólidos disueltos, alcalinidad, dureza, DQO, bicarbonato, magnesio, sodio, potasio y cloruros, y de forma negativa turbidez, sólidos en suspensión, DBO, nitrato, nitrito y amonio. Las variables más correlacionadas con el segundo componente de manera positiva fueron oxígeno disuelto, $\mathrm{pH}$, carbonato y sodio, y de forma negativa dureza, DBO, bicarbonato, potasio, sulfato, nitrato, nitrito, amonio y sulfato. Respecto del tercer componente se correlacionaron positivamente temperatura, $\mathrm{pH}$, dureza, carbonato, calcio, sulfato, nitrato y nitrito, en tanto que de forma negativa la turbidez, sólidos en suspensión y amonio. De lo anterior puede inferirse que los tres componentes presentados agrupan a los sitios de muestreo en función de su contenido de

CUADRO III. MATRIZ DE AUTOVECTORES QUE MUESTRA LA CONTRIBUCIÓN DE LOS CARACTERES FÍSICOS Y QUÍMICOS A LOS TRES PRIMEROS COMPONENTES DEL ANÁLISIS DE COMPONENTES PRINCIPALES REALIZADO.

\begin{tabular}{lccc}
\hline & \multicolumn{3}{c}{ Componente } \\
\cline { 2 - 4 } Variables & 1 & 2 & 3 \\
\hline Temperatura & 0.04 & 0.08 & 0.45 \\
Oxígeno disuelto & 0.07 & 0.36 & -0.11 \\
pH & 0.06 & 0.31 & 0.31 \\
Conductividad & 0.3 & -0.03 & 0.12 \\
Turbidez & -0.19 & -0.08 & -0.17 \\
Sólidos totales & 0.31 & -0.08 & -0.03 \\
Sólidos en suspensión & -0.17 & -0.07 & -0.17 \\
Sólidos disueltos & 0.31 & $1.60 \mathrm{E}-03$ & -0.08 \\
Alcalinidad & 0.3 & -0.1 & $7.00 \mathrm{E}-02$ \\
Dureza & 0.24 & -0.22 & 0.17 \\
DBO & -0.2 & -0.19 & 0.09 \\
DQO & 0.27 & -0.07 & -0.06 \\
Carbonato & 0.03 & 0.28 & 0.35 \\
Bicarbonato & 0.2 & -0.27 & -0.2 \\
Calcio & -0.01 & -0.12 & 0.2 \\
Magnesio & 0.23 & -0.13 & 0.03 \\
Sodio & 0.17 & 0.33 & -0.05 \\
Potasio & 0.27 & -0.19 & 0.08 \\
Cloruro & 0.31 & 0.01 & -0.02 \\
Sulfato & -0.05 & -0.29 & 0.34 \\
Nitrato & -0.17 & -0.19 & 0.35 \\
Nitrito & -0.18 & -0.23 & 0.25 \\
Amonio & -0.14 & -0.17 & -0.16 \\
Fosfato & 0.06 & -0.35 & -0.12 \\
\hline
\end{tabular}

DBO: demanda biológica de oxígeno, DQO: demanda química de oxígeno. sales y de materia orgánica, ya que éstas fueron las variables de mayor peso en su construcción. De esta manera quedan en evidencia los problemas que pueden ocasionar las descargas de los efluentes pluviales en los cuerpos de agua estudiados.

De acuerdo con el PCA realizado, las principales diferencias entre ambas secciones de la LMG se reflejaron en mayores valores de conductividad (superiores a los $3000 \mu \mathrm{S} / \mathrm{cm}$ en Sec2), lo que también se reflejó en mayor presencia de sólidos totales y disueltos, encontrándose que el ion de mayor concentración fue el cloruro. La Sec2 también se diferenció de la Sec1 por tener mayores valores de materia orgánica en sus aguas, determinadas como DQO, $\mathrm{pH}$ y oxígeno disuelto, y menores valores de DBO, nitratos, sólidos en suspensión y turbidez. Estas diferencias entre secciones también se observaron estacionalmente en cada sección: en la Sec2 fueron más notorias en las estaciones de verano y otoño, cuando el contenido de sales (conductividad, sólidos totales y disueltos y cloruros) y de materia orgánica determinada como DQO tienden a aumentar, mientras que en la Sec1 estas diferencias estacionales fueron poco acentuadas.

\section{Caracterización microbiológica}

En concordancia con la caracterización física y química presentada, la posible contaminación por materia orgánica observada en la LMG también se evidencia en los conteos de microorganismos realizados (Cuadro II). La mayor presencia de microorganismos potencialmente patógenos se observó en primavera, con presencia de coliformes totales, fecales y enterococos en prácticamente todas las estaciones del año. Los valores máximos de coliformes totales se observaron en primavera y otoño con promedios de $522 \pm 491$ y $197 \pm 365 \mathrm{NMP} / 100 \mathrm{~mL}$ (p $<0.05$ ), respectivamente, y con valores mínimos de $<$ 3 y máximos de $1100 \mathrm{NMP} / 100 \mathrm{~mL}$ para ambas estaciones del año. La totalidad de los valores máximos encontrados correspondieron a los sitios de muestreo M3 y M4 de la Sec2 y M7 y M8 de la Sec1. Por otro lado, los valores máximos de coliformes fecales se observaron en primavera con valores promedio de $88 \pm 181$, mínimos de $<3 \mathrm{NMP} / 100 \mathrm{~mL}$ y máximos de $500 \mathrm{NMP} / 100 \mathrm{~mL}(\mathrm{p}<0.05)$, siendo la Sec1 la principal receptora de la totalidad de los valores más altos. Los valores máximos de enterococos también se registraron en primavera, observándose de 150 y de $500 \mathrm{NMP} / 100 \mathrm{~mL}$ para Sec1 y Sec2, respectivamente $(\mathrm{p}<0.05)$. Anteriormente se ha informado que las aguas recreativas pueden albergar microorganismos indicadores (Pachepsky et al. 2016) que podrían 
causar trastornos gastrointestinales, de la piel, de los ojos e infecciones del oído por contacto en actividades recreativas (Andraus et al. 2014, Sabino et al. 2014). Los organismos indicadores son bacterias que se utilizan como señales de calidad/condición higiénica en el medio ambiente. Para determinar la posible presencia de patógenos entéricos en este tipo de ambientes, los organismos indicadores son actualmente el estándar funcional, dada su asociación con la contaminación fecal reciente (NRC 2004, Harwood et al. 2018). Sobreviven sólo por cortos periodos de tiempo en el agua (Rubio-Arias et al. 2016), por lo que sirven de alerta sobre la posible presencia de patógenos potenciales (WHO 2003, Gracia et al. 2018). La alta prevalencia de especies de enterococos puede ser un indicador de posibles riesgos para la salud de los usuarios que frecuentan estas aguas (Adeniji et al. 2019). Nevers et al. (2018) estudiaron la densidad de Escherichia coli en aguas del lago Michigan (Indiana), concluyendo que el principal aporte de este microorganismo es por aves, seguido de perros y humanos. Byappanahalli et al. (2015) reportaron la necesidad de diferenciar por métodos moleculares su procedencia y así delinear adecuados métodos de tratamiento. E. coli fue detectada en la LMG en mayor magnitud $(\mathrm{p}<0.05)$ también en primavera $(11.38 \pm 24.8 \mathrm{NMP} / 100 \mathrm{~mL})$, con valores máximos de $70 \mathrm{NMP} / 100 \mathrm{~mL}$ en la Sec1. Se observó una gran diversidad de aves en la totalidad de la superficie de la laguna en todas las instancias de muestreo realizadas, lo cual, sumado a la presencia de perros proveniente de las inmediaciones de ésta, proporcionan las condiciones para contar con diferentes posibles fuentes de procedencia.

\section{Contaminantes específicos}

Ninguno de los contaminantes específicos investigados en las muestras de agua fue detectado(Cuadro II). En el caso de los sedimentos estudiados, no se detectaron HTP, cadmio, mercurio ni selenio (Cuadro IV). Por otro lado, en los sedimentos de LMG se detectaron concentraciones de arsénico, bario, cinc, cobre, cromo, níquel, plata y plomo inferiores a los límites de referencia propuestos por las normas canadienses sobre la calidad de los sedimentos para la protección de la vida acuática para aguas naturales dulces (CCME 2014) y por las normas australianas y neozelandesas para la calidad del agua dulce y salada (ANZECC 2000).

Los metales pesados son especialmente de alto riesgo para el ambiente dado su tiempo largo de persistencia en la naturaleza y su posible bioacumulación y biomagnificación (Sandeep et al. 2019). Elementos
CUADRO IV. DETERMINACIONES ANALÍTICAS REALIZADAS EN LAS MUESTRAS DE SEDIMENTOS RECOLECTADAS*

\begin{tabular}{cc}
\hline Parámetro & Concentración $(\mathrm{mg} / \mathrm{kg})$ \\
\hline HTP & $<20$ \\
Arsénico & $0.91 \pm 0.17$ \\
Bario & $418 \pm 56$ \\
Cadmio & $<1$ \\
Cinc & $65 \pm 13$ \\
Cobre & $14 \pm 2$ \\
Cromo & $4.4 \pm 6.2$ \\
Mercurio & $<0.2$ \\
Níquel & $16.4 \pm 1.5$ \\
Plata & $1.33 \pm 0.30$ \\
Plomo & $3.20 \pm 5.97$ \\
Selenio & $<0.8$ \\
\hline
\end{tabular}

Debido a la poca variabilidad de los resultados obtenidos para cada analito $(\mathrm{p}>0.05)$, se presenta de forma representativa el promedio con su desviación estándar de todos los valores obtenidos en todas las muestras de sedimento recolectadas en el estudio. HTP: hidrocarburos totales del petróleo.

como $\mathrm{Cu}, \mathrm{Zn}, \mathrm{Co}, \mathrm{Fe}$ no pueden considerarse un peligro para la calidad del agua a menos que alcancen altas concentraciones, ya que son necesarios para la vida animal (Vardhan et al. 2019). En contraste, Cd, $\mathrm{Hg}$ y $\mathrm{Pb}$ siempre se comportan como tóxicos en los organismos (Qingjie et al. 2008). Debido a que los sedimentos concentran los metales y la concentración de estos elementos en los sedimentos es menos variable que en el agua, estos son adecuados para monitorear el depósito de metales a largo plazo en los ecosistemas. Si bien en la LMG no se detectaron cantidades de estos compuestos superiores a las propuestas en la bibliografía consultada, es importante su conocimiento para monitorear su evolución en función del tiempo y desarrollar un adecuado control de su calidad.

\section{Índices de calidad ambiental y evaluación de posibles usos}

Todo lo expuesto sobre la caracterización del agua se resume en el ICA calculado, ya que los valores observados durante el tiempo de estudio no presentaron diferencias significativas $(\mathrm{p}>0.05)$ y clasifican a las aguas de la LMG en la categoría de calidad media. Los valores del índice ICOMO obtenidos indican un aporte de materia orgánica baja y esporádico durante el año (Fig. 3), que adquiere valores con diferencias significativas $(\mathrm{p}<0.05)$ en primavera/otoño $(\sim 0.10)$ respecto de verano/invierno $(\sim 0.23)$.

La legislación argentina clasifica las aguas recreacionales en tres categorías de acuerdo con la 


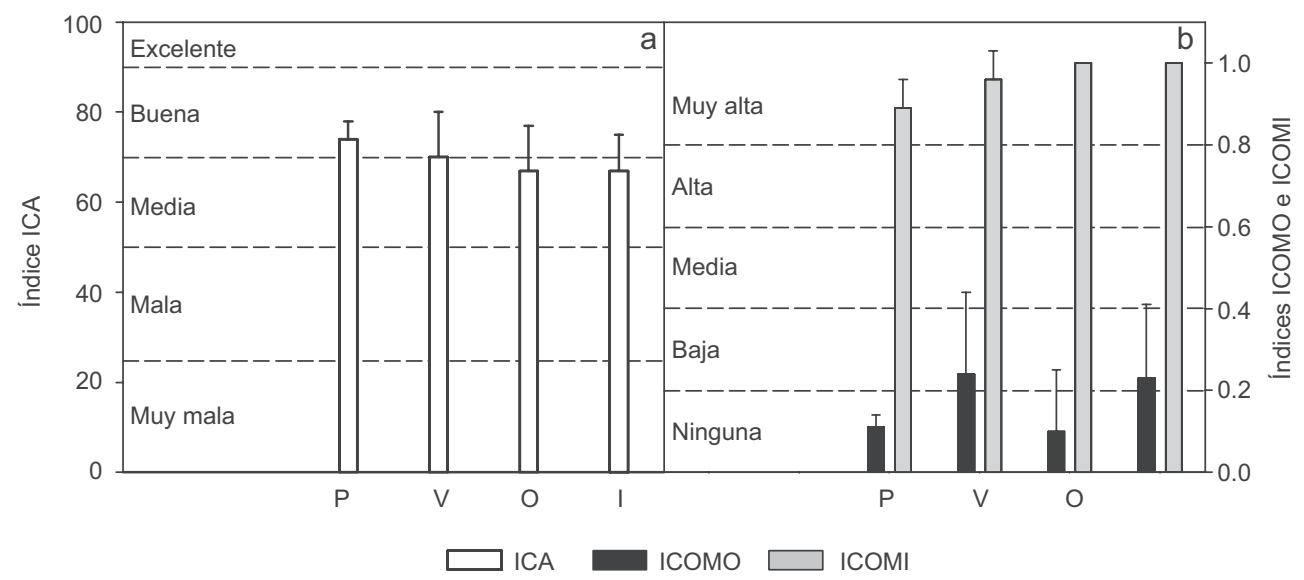

Fig. 3. Diferentes índices de calidad del agua calculados a partir de los estudios realizados en la laguna María la Gorda durante las diferentes estaciones del año. ICA: índice de calidad del agua, ICOMO: índice de contaminación por materia orgánica, ICOMI: índice de contaminación por mineralización.

forma en que se desarrollan las actividades: $i$ ) con contacto directo, $i$ i) sin contacto directo y iii) pasivas. Las actividades con contacto directo son aquellas en las que se produce inmersión del cuerpo; las que no tienen contacto directo son aquellas en que existe un contacto eventual con el agua, y las pasivas son las que apuntan sólo al disfrute estético. De esta forma, la calidad del agua de la LMG cumple con los parámetros establecidos de oxígeno disuelto, amonio, E. coli, fósforo, cromo, plomo, cadmio, mercurio y arsénico para las tres categorías establecidas. Sin embargo, para los parámetros $\mathrm{DBO}$, nitrato y $\mathrm{pH}$, la Sec1 de la LMG no cumple con lo regulado por esta legislación, la cual establece valores de DBO de 3,10 y $15 \mathrm{mg} / \mathrm{L}$ para las categorías $i$, ii y iii, respectivamente, y de nitratos $<10 \mathrm{mg} / \mathrm{L}$ con $\mathrm{pH}$ de 6 a 9 para todas las categorías. Las aguas contaminadas con nitratos pueden ser perjudiciales para la salud humana en general, ya que su consumo puede producir nitrosaminas por la acción de las bacterias intestinales, pudiendo ser estos compuestos potenciales cancerígenos (WHO 2003). Por otro lado, se ha detectado que los nitratos son perjudiciales para los niños, sobre todo para los lactantes, ya que al reducirse a nitritos pueden provocar la enfermedad conocida como metahemoglobinemia, la que en muchos casos puede tener consecuencias fatales (de Miguel-Fermández y Vázquez-Taset 2006).

Otro uso potencial de estas aguas es su utilización para riego, ya que al estar la LMG catalogada como reserva urbana, una de las actividades a desarrollar es la de forestación y acondicionamiento de sus alrededores para su adecuado mantenimiento estético. Sin embargo, los resultados obtenidos demuestran que las características del agua de la LMG, cuyo $\mathrm{pH}$ estacional es cercano o superior a 9 y su conductividad de aproximadamente 2000 a 3800 $\mu \mathrm{S} / \mathrm{cm}$, no son adecuadas para el riego (Fig. 4a). A partir del diagrama de Piper realizado (Fig. 4b) se observa que los iones principales en su composición química son el cloruro y el sodio, por lo que se clasifica como clorurada sódica. De acuerdo con la clasificación de Riverside (US Salinity Lab Staff 1954), el agua de la LMG se incluye en la categoría S4C4, es decir, con un alto contenido de sodio y excesiva salinidad, por lo que es de muy mala calidad para ser utilizada en riego (Avellaneda et al. 2004). Esta elevada mineralización se observa también en el índice ICOMI calculado (Fig. 3b), el cual mostró valores muy cercanos o iguales a la unidad durante todo el año de estudio, no encontrándose diferencias significativas entre éstos ( $\mathrm{p}>0.05)$. De acuerdo con lo propuesto por Ayres y Westcot (1994), la calidad del agua de la LMG se considera como de riego moderado a severo para su uso en riego. Misaghi et al. (2017) estudiaron la calidad del agua para riego del río Ghezel Ozan en Irán, determinando que la misma varía de forma estacional, lo cual atribuyeron a la variación de parámetros como el $\mathrm{pH}$ con las condiciones climáticas. Lothrop et al. (2018) relacionaron estos cambios temporales de la calidad del agua de fuentes naturales para riego, con posibles eventos esporádicos de contaminación. Esta situación puede ser la causante de las mencionadas fluctuaciones en el agua de la LMG durante el año de estudio, resultado de 


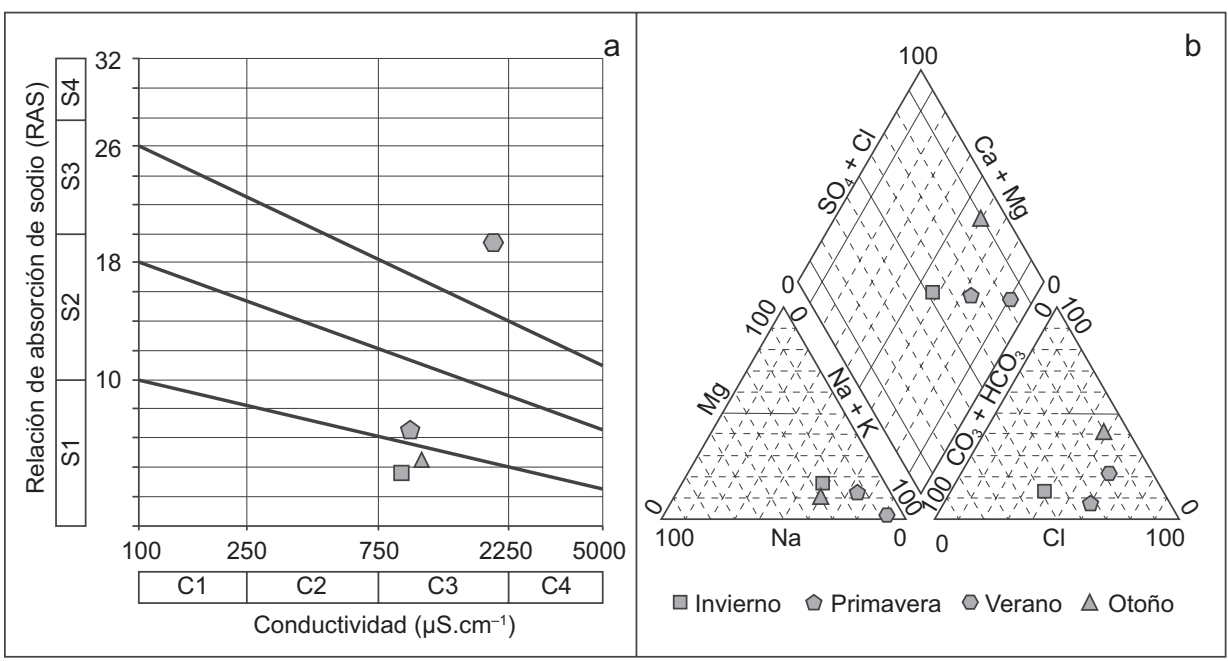

Fig. 4. Diagramas (a) de Riverside y (b) de Piper elaborados para las muestras de agua recolectadas en las diferentes estaciones del año.

la desembocadura de canales pluviales en la laguna.

De todo lo expuesto se puede concluir que el agua de la LMG tiene una calidad ambiental media, siendo de cuidado su uso con fines recreativos debido a la presencia de contaminación por materia orgánica. La demostración de la baja calidad de este espacio, catalogado como reserva urbana, obliga a la implementación de medidas tendientes al saneamiento y mantenimiento de la buena calidad ambiental de la zona de estudio. Esto, con la finalidad de darle el uso para el cual está destinada sin que en el futuro surjan inconvenientes ambientales que lleven a la aparición de problemas de salud en la población de Río Gallegos, asociados con la problemática planteada.

\section{AGRADECIMIENTOS}

Los autores agradecen la contribución financiera de Universidad Tecnológica Nacional, Facultad Regional Santa Cruz, mediante el proyecto de investigación INN4910 "Caracterización ambiental de Reservas Naturales Urbanas de la ciudad de Río Gallegos: Laguna María la Gorda y Laguna de Los Patos".

\section{REFERENCIAS}

Adeniji O.O., Sibanda T. y Okoh A.I. (2019). Recreational water quality status of the Kidd's Beach as determined by its physicochemical and bacteriological quality parameters. Heliyon 5, e01893. https://doi.org/10.1016/j. heliyon.2019.e01893
Allan J.D. y Castillo M.M. (2007). Stream ecology: Structure and function of running waters. Springer, Países Bajos, 436 pp. https://doi.org/10.1007/978-14020-5583-6

Andraus S., Pimentel I.C. y Dionisio J.A. (2014). Microbiological monitoring of seawater and sand of beaches Matinhos, Caiobá e Guaratuba-PR, Brazil. Estud. Biol. 36 (86), 43-55. https://doi.org/10.7213/estud. biol.36.086.AO05

ANZECC (2000). Australian and New Zealand guidelines for fresh and marine water quality. Vol. 1. The guidelines. Manual. Australian and New Zealand Environment and Conservation Council. Australia, 314 pp.

Avellaneda M.O., Bermejillo A.I. y Mastrantonio L.E. (2004). Aguas de riego, calidad y evaluación de su factibilidad de uso. EDIUNC, Mendoza, Argentina, $143 \mathrm{pp}$.

Ayers R. y Westcot D. (1994). Water quality for agriculture. FAO Irrigation and Drainage Paper 29, rev. 1. Food and Agriculture Organization of the United Nations, Roma, Italia.

Byappanahalli M.N., Nevers M.B., Whitman R.L., Ge Z., Shively D., Spoljaric A. y Przybyla-Kelly K. (2015). Wildlife, urban inputs, and landscape configuration are responsible for degraded swimming water quality at an embayed beach. J. Great Lakes Res. 41 (1), 156-163. https://doi.org/10.1016/j.jglr.2014.11.027

CCME (2014). Canadian environmental quality guidelines. Manual. Canadian Council of Ministers of the Environment. Canadá, 215 pp.

Decristófaro L., Ferrari S. y Albrieu C. (2014). Reservas naturales urbanas en el sur de Santa Cruz (Argentina): importancia, caracterización del uso público e 
incidencia. Informes Científicos Técnicos-UNPA 6 (2), 90-117. http://doi.org/10.22305/ict-unpa.v6i2.96

De Miguel-Fernández C. y Vázquez-Taset Y.M. (2006). Origen de los nitratos $\left(\mathrm{NO}_{3}\right)$ y nitritos $\left(\mathrm{NO}_{2}\right)$ y su influencia en la potabilidad de las aguas subterráneas. Minería y Geología 22 (3), 1-9.

Elordi M.L., Colman Lerner J.E. y Porta A.A. (2013). Estimación y evaluación de la calidad del agua de los arroyos las Piedras y San Francisco mediante el índice de calidad del agua WQI-NSF. En: Contaminación atmosférica e hídrica en Argentina (Puliafitto, Allende, Panigatti, Eds.). UTN, Mendoza, Argentina, pp. 207-216.

EPA (2013). Integrated water quality report 2012 Monaghan and Louth. Manual. Environmental Protection Agency, Irlanda, 80 pp.

Ferrari S., Decristófaro L., Estrampes L. y Albrieu C. (2014). Reservas naturales urbanas: herramientas para fomentar el turismo y la recreación en comunidades del sur de Santa Cruz (Argentina). Memorias del. VI Congreso latinoamericano de investigación turística. Neuquén, Argentina, 24 al 26 de septiembre, 1-17.

Ferrari S. y Montero G. (2015). Plan de manejo del sistema de reservas naturales urbanas de la ciudad de Río Gallegos. Municipalidad de Río Gallegos, Argentina, 195 pp.

Gracia D.S., Cope J.R., Roberts V.A., Cikesh B.L., Kahler A.M., Vigar M., Hilborn E.D., Wade T.J., Backer L.C., Montgomery S.P. y Secor W.E. (2018). Outbreaks associated with untreated recreational water - United States, 2000-2014. MMWR Morb. Mortal. Wkly. Rep. 67 (25), 701-706. https://doi.org/10.15585/mmwr.mm6725a1

Hammer O, Harper DAT y Ryan PD. (2001). PAST: Paleontological statistics software package for education and data analysis. Palaeontol. Electronica 4, 1-9.

Harwood V.J., Korajkic A., Verbyla M., Iriarte M. y Shanks O. (2018). General and host-associated bacterial indicators of fecal pollution. En: Sanitation and disease in the 21st century (Rose J.B. y Jiménez-Cisneros B, Eds.). Global Water Pathogen Project. UNESCOIntergovernmental Hydrological Programme-Michigan State University, 1-38.

Lothrop N., Bright K.R., Sexton J., Pearce-Walker J., Reynolds K.A. y Verhougstraete M.P. (2018). Optimal strategies for monitoring irrigation water quality. Agric. Water Manag. 199, 86-92. https://doi. org/10.1016/j.agwat.2017.12.018

McCutcheon S.C., Martin J.L. y Barnwell T.O. (1993). Water quality. En: Handbook of hydrology (Maidment D.R., Ed.). McGraw-Hill, USA.

Misaghi F., Delgosha F., Razzaghmanesh M. y Myers B. (2017). Introducing a water quality index for assessing water for irrigation purposes: A case study of the Ghezel Ozan River. Sci. Total Environ. 589, 107-116. https://doi.org/10.1016/j.scitotenv.2017.02.226
Nevers M.B., Byappanahalli M.N., Shively D., Buszka P.M., Jackson P.R. y Phanikumar M.S. (2018). Identifying and eliminating sources of recreational water quality degradation along an urban coast. J. Environ. Qual. 47, 1042-1050. https://doi.org/10.2134/ jeq2017.11.0461

NRC (2004). Indicators for waterborne pathogens. Manual. National Research Council. Washington, DC, EUA. https://doi.org/10.17226/11010

Pachepsky Y., Shelton D., Dorner S. y Whelan G. (2016). Can E. coli or thermotolerant coliform concentrations predict pathogen presence or prevalence in irrigation waters? Crit. Rev. Microbiol. 42 (3), 384-393. https:// doi.org/10.3109/1040841X.2014.954524

Qingjie G., Jun D., Yunchuan X., Qingfei W. y Liqiang Y. (2008). Calculating pollution indices by heavy metals in ecological geochemistry assessment and a case study in parks of Beijing. J. China Univer. Geosci. 19 (3), 230241. https://doi.org/10.1016/S1002-0705(08)60042-4

Ramírez A., Restrepo R. y Viña G. (1997). Cuatro índices de contaminación para caracterización de aguas continentales. Formulaciones y aplicaciones. Cienc. Tecnol. Futuro. 1 (3), 135-153.

Rice E.W., Baird R.B. y Eaton A.D. (2017). Standard methods for the examination of water and wastewater. 23th ed. American Public Health Association (AWWA), Water Environment Federation, Washington, DC.

Rubio-Arias H.O., Rey-Burciaga N.I., Quintana R.M., Ochoa-Rivero J.M., Saucedo-Terán R.A. y Ortiz-Delgado R.C. (2016). Recreational water quality index (RWQI) for Colina Lake in Chihuahua, Mexico. Acta Universitaria 26 (3), 14-22. https://doi.org/1a0.15174/au.2016.901

Sabino R., Rodrigues R., Costa I., Carneiro C., Cunha M., Duarte A., Faria N., Ferreira F.C., Gargaté M.J., Júlio C., Martins M.L., Nevers M.B., Oleastro M., Solo-Gabriele H., Veríssimo C., Viegas C., Whitman R.L. y Brandao J. (2014). Routine screening of harmful microorganisms in beach sands: implications to public health. Sci. Total Environ. 472, 1062-1069. https://doi. org/10.1016/j.scitotenv.2013.11.091

Sandeep K., Shiv P., Krishna K.Y., Manoj S., Neha G., Shivani N., Quang-Vu B., Hesam K., Shakeel A.K., Sunita Y. y Lal C.M. (2019). Hazardous heavy metals contamination of vegetables and food chain: Role of sustainable remediation approaches - A review. Environ. Res. 179, 108792. https://doi.org/10.1016/j. envres.2019.108792

US-EPA (1978). Method 418.1. Petroleum hydrocarbons, total recoverable (spectrophotometric, infrared). United States Environmental Protection Agency. Analytical method, Washington, D.C., EUA, 8 pp.

US-EPA (1992). Method 7061A. Arsenic (atomic absorption, gaseous hydride). United States Environmental 
Protection Agency. Analytical method, Washington, D.C., EUA, 6 pp.

US-EPA (1994). Method 7741A. Selenium (atomic absortion, gaseous hydride). United States Environmental Protection Agency. Analytical method, Washington, D.C., EUA, 5 pp.

US-EPA (1996). Method 3050B. Acid digestion of sediments, sludges and soils. United States Environmental Protection Agency. Analytical method, Washington, D.C., EUA, 12 pp.

US-EPA (2007). Method 7000B. Flame atomic absorption apectrophotometry. United States Environmental Protection Agency. Analytical method, Washington, D.C., EUA, 23 pp.

US-EPA (2007). Method 3550C. Ultrasonic extraction. United States Environmental Protection Agency. Analytical method, Washington, D.C., EUA, 17 pp.
US-EPA (2012). EPA 820-F12-058. Recreational water quality criteria. Office of Research and Development., United States Environmental Protection Agency. Manual, Washington, D.C., EUA, 69 pp.

US Salinity Lab Staff (1954). Diagnosis and improvement of saline and alkali soils. Handbook 60. United States Department of Agriculture, $160 \mathrm{pp}$.

Vardhan K.H., Kumar P.S. y Panda R.C. (2019). A review on heavy metal pollution, toxicity and remedial measures: Current trends and future perspectives. J. Mol. Liq. 290, 111197. https://doi.org/10.1016/j.molliq.2019.111197

Weiner E.R. (2012). Applications of environmental aquatic chemistry, a practical guide. 3rd ed. CRC Press, USA, $618 \mathrm{pp}$.

WHO (2003). Guidelines for safe recreational-water environments. Coastal and fresh water. Vol. 1. World Health Organization, Ginebra, 125 pp. 